\title{
KKT Proximity Measure Versus Augmented Achievement Scalarization Function
}

\author{
Mohamed Abouhawwash \\ Mansoura University \\ Department of Mathematics \\ Faculty of Science
}

\author{
M. A. Jameel \\ Sana'a University \\ Department of Mathematics \\ Faculty of Education, Arts and Sciences - Khawlan
}

\begin{abstract}
KKT proximity measure (KKTPM) is use as metric for obtained how we are close to the from a corresponding Pareto-optimal (PO) point without any knowledge about the true optimum point. This metric use one such common a scalarization method that also guarantees to find any PO solution that is achievement scalarizing function (ASF) method. Since that KKTPM formulation is based on augmented achievement scalarizing function (AASF) to avoid weak PO solutions. This paper studies a relation between KKTPM values and AASF values. Aim of this study to know the advantage and disadvantage of both measures. Also, this paper discusses some special cases to know the merits of both measures and to confirm that KKT proximity measure is an essential measure for convergence. In addition, this study investigates the correlation plot between these two measures for ZDT test problems, results show the difference in values and therefore cannot obtain a perfect correlation between KKTPM values and AASF values. Hence, it can be said that KKT proximity measure is better.
\end{abstract}

\section{Keywords}

Multi-objective optimization, Exact KKT proximity measure, Direct KKT proximity measure, AASF approach

\section{INTRODUCTION}

Recently, a number of evolutionary algorithms have been proposed that attempt to find a set of PO solutions for a problem. This type of solutions should be as close as possible to the Pareto front and, at the same time, it should be as diverse as possible on the Pareto front. Most of these algorithms have no evidence of convergence. It is therefore necessary to have performance metrics to evaluate this type of evolutionary algorithms. For this purpose, Deb and Abouhawwash recently in [7] have proposed KKTPM metric that is able to determine relative closeness for any solution (in objective space) from the theoretical optimum solution without actually knowing the location of the true optimal solution. This metric depends on KKT optimality conditions that guarantee to be satisfied at only optimal solutions. This means that for multi-objective optimization problems (MOPs) must satisfy all KKT optimality conditions at an optimum solution (or KKT point) [13], [5], [17], [18], [4]. The proposal of Deb and Abouhawwash called exact (or optimal) KKT proximity metric and the value of this metric called exact (or optimal) KKTPM value. Exact KKTPM has generalized the idea of approximate KKT point definition which proposed in an earlier study for single-objective optimization problems (SOPs) [10 12], [2 19] to be applied to MOPs. The KKTPM procedure can be applied also to MOP with conflicting objectives. The exact KKTPM computational procedure requires the first-order gradient for both objectives and constraints functions at all point thereby making the exact procedure computationally expensive and therefore extremely time-consuming for any practical use.

To tackle this shortcoming, the authors in [8] proposed several alternative approximate methods so that KKTPM value can be computed in a computationally fast method. This metric called Direct KKT proximity metric and the value of this metric called direct KKTPM value. One of the most important advantages of these metrics is that they do not require prior knowledge of the true PO solutions, while the previously proposed performance metrics require prior knowledge of this type of solution, such as hypervolume measure [21], [3], inverse generational distance (IGD) metric [9], and other metrics [6], [20], [14], [11].

Both of the suggested KKTPM above [7], [8] used one of the popular ways for handling MOPs, and also its guarantees to find any PO solution. This method is called achievement scalarizing function (ASF) [22]. This method requires two parameters (reference point (utopian) and a weight vector).

Since KKTPM formulation is based on augmented ASF approach, then, this paper studies a correlation plot between both exact KKTPM values and AASF values, also between direct KKTPM values and AASF values. The aim of this study if there a perfect correlation, AASF value can be used as a metric (i.e., can be found out how close any point of Pareto front is through the AASF value at that point) and no need for KKTPM metric. This study shows the importance of KKTPM as a measure that ensures that each PO solution has a zero value, and there is no way of telling whether a point is PO solution from the AASF or ASF value. In other words, The study demonstrates that the KKTPM is an essential metric of convergence and the AASF approach cannot be used as a convergence metric.

In the remainder of this paper. In Section 2 the augmented ASF approach is presented only briefly. In Section 3. KKTPM computation procedures (Exact and Direct Methods) are summarized. Special cases for KKT proximity measure are discussed in Section 4 and, in the same section, a correlation plot between KKTPM values and AASF values for ZDT test problems [23] is investigated. Finally, the conclusions are presented in Section 5 


\section{AUGMENTED ACHIEVEMENT SCALARIZING FUNCTION (AASF) APPROACH}

Let us consider a $n$-variable, and $M$-objective optimization problem in its form:

$$
\begin{array}{rlr}
\operatorname{Minimize}_{(\mathbf{x})} & f_{m}(\mathbf{x}), & m=1,2, \ldots, M, \\
\text { subject to } & g_{j}(\mathbf{x}) \leq 0, & j=1,2, \ldots, J .
\end{array}
$$

The achievement scalarizing function (ASF) one of the most widely used methods of dealing with above problem. This method was originally suggested by Wierzbicki [22]. The ASF procedure requires reference point $\mathbf{z}$ ( $\mathbf{z}^{\text {ideal }}$ or utopian) and weight $\mathbf{w}$ as parameters, the ASF problem is described as follows:

$$
\begin{aligned}
\operatorname{Minimize}_{(\mathbf{x})} & A S F(\mathbf{x}, \mathbf{z}, \mathbf{w})=\max _{i=1}^{M}\left(\frac{f_{i}(\mathbf{x})-z_{i}}{w_{i}}\right), \\
\text { subject to } & g_{j}(\mathbf{x}) \leq 0, \quad j=1,2, \ldots, J .
\end{aligned}
$$

For illustrating the working principle of the ASF procedure for finding PO solution in [7]. Any Pareto-optimal or properly/weakly PO solution can be generated by the above ASF minimization process. But it turns out that in case weakly PO solution, ASF procedure computed from the utopian (or ideal) point may result in a weak PO solution as well. For discarding weakly PO solution, the following AASF approach was suggested [15]:

$$
\begin{array}{rl}
\operatorname{Minimize}_{(\mathbf{x})} \quad A & A S F(\mathbf{x}, \mathbf{z}, \mathbf{w})=\max _{i=1}^{M}\left(\frac{f_{i}(\mathbf{x})-z_{i}}{w_{i}}\right) \\
+ & \rho \sum_{i=1}^{M}\left(\frac{f_{i}(\mathbf{x})-z_{i}}{w_{i}}\right),
\end{array}
$$$$
\text { Subject to } g_{j}(\mathbf{x}) \leq 0, \quad j=1,2, \ldots, J .
$$

Here, $\rho>0$ takes a small value $\left(\approx 10^{-4}\right)$. Where the additional term is added to avoid finding weak points. Figure 1 shows how the working of the AASF approach. The additional term makes the isoAASF lines inclined to objective axes (see Figure 11). For the example shown, intersect both of weight vector and weakly PO front at point $\mathbf{A}$, but at this point, the AASF value is not the minimum value required. The point $\mathbf{O}$ has a smaller AASF value as the corresponding iso-AASF lines intersect the weight vector closer to the reference point $\mathbf{z}$. A bit of reflection will reveal that with a positive value $\rho$, the optimal solution for the previous AASF problem is the point $\mathbf{O}$. The AASF has a larger value at a point $\mathbf{A}$ or other weak PO points because it conforms to the w-line at a higher point. Interestingly, the value of both AASF and ASF at strictly PO point $\mathrm{O}$ is identical. More information about ASF and AASF approaches in [7 [15]. The advantage of ASF is that any (weakly) PO solution can be obtained by only moving the reference point [15. 16]. This means that augmented ASF or ASF value depends on the location of the reference point $\mathbf{z}$

In the following section, the KKTPM (exact and direct) computation procedures on based AASF are briefly summarized, and the most important properties of KKT proximity measure are presented.

\section{KKTPM COMPUTATION PROCEDURE (EXACT AND DIRECT METHODS)}

As mentioned in Section 11 Deb and Abouhawwash in [7], [8] defined two methods for an approximate KKT solution to calculate KKTPM value for any iterate $\mathbf{x}^{k}$. Exact and direct KKTPM metrics have used for an estimate of the proximity of any point from a

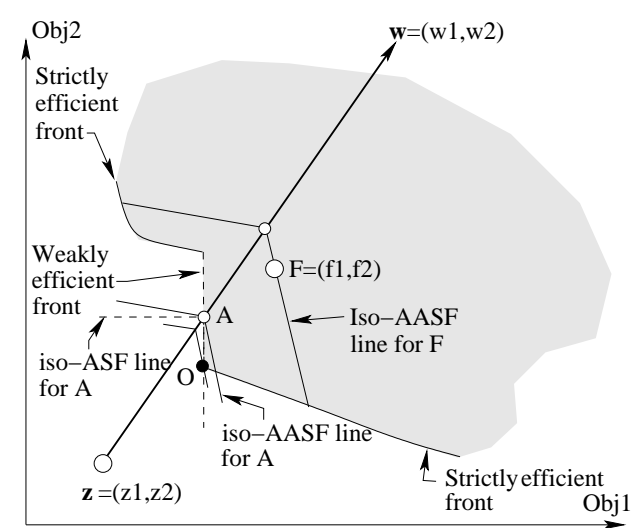

Fig. 1: AASF approach is illustrated.

corresponding PO point without really knowing the exact location of the optimum point. These metrics used ASF approach and which its guarantees to find any PO solution. Since the problem stated in Equation 2 is non-differentiable, the authors in [7], [8] used the following formula:

$$
\begin{aligned}
\operatorname{Min}_{\left(\mathbf{x}, x_{n}+1\right)} & F\left(\mathbf{x}, x_{n+1}\right)=x_{n+1} \\
\text { subject to } & \left(\frac{f_{i}(\mathbf{x})-z_{i}}{w_{i}}\right)-x_{n+1} \leq 0, \quad j=1,2, \ldots, M . \\
& g_{j}(\mathbf{x}) \leq 0, \quad j=1,2, \ldots, J
\end{aligned}
$$

Here, the new variable $x_{n+1}$ is added to $M$ number of inequality constraints to make the problem smooth. To ensure finding a strictly PO point, the authors used AASF approach as follows:

$$
\begin{aligned}
\operatorname{Min}_{\left(\mathbf{x}, x_{n}+1\right)} \quad F\left(\mathbf{x}, x_{n+1}\right)=x_{n+1}, \\
\text { s.t. } \quad\left(\frac{f_{i}(\mathbf{x})-z_{i}}{w_{i}}\right)+\rho \sum_{i=1}^{M}\left(\frac{f_{i}(\mathbf{x})-z_{i}}{w_{i}}\right)-x_{n+1} \leq 0, \forall i \\
g_{j}(\mathbf{x}) \leq 0, \quad j=1,2, \ldots, J .
\end{aligned}
$$

Since a slack variable $x_{n+1}$ for this problem will be satisfied by setting:

$$
x_{n+1}=\max _{i=1}^{M}\left(\frac{f_{i}(\mathbf{x})-z_{i}}{w_{i}}\right)+\rho \sum_{i=1}^{M}\left(\frac{f_{i}(\mathbf{x})-z_{i}}{w_{i}}\right) .
$$

The weight value can be computed for the $i$-th objective as follows:

$$
w_{i}=\frac{f_{i}(\mathbf{x})-z_{i}}{\sqrt{\sum_{k=1}^{M}\left(f_{k}(\mathbf{x})-z_{k}\right)^{2}}} .
$$

The exact KKTPM problem for a smooth objective function $\mathbf{y}=$ $\left(\mathbf{x} ; x_{n+1}\right)$ given by Equation 5 can be written as follows:

$$
\begin{aligned}
{\text { Min. }\left(\epsilon_{k}, x_{n+1}, \mathbf{u}\right)} & \epsilon_{k}+\sum_{j=1}^{J}\left(u_{M+j} g_{j}\left(\mathbf{x}^{k}\right)\right)^{2}, \\
\text { S.t. } & \left\|\nabla F(\mathbf{y})+\sum_{j=1}^{M+J} u_{j} \nabla G_{j}(\mathbf{y})\right\|^{2} \leq \epsilon_{k}, \\
& \sum_{j=1}^{M+J} u_{j} G_{j}(\mathbf{y}) \geq-\epsilon_{k}, \\
& u_{j} \geq 0, j=1,2, \ldots,(M+J), \\
& -x_{n+1} \leq 0 .
\end{aligned}
$$




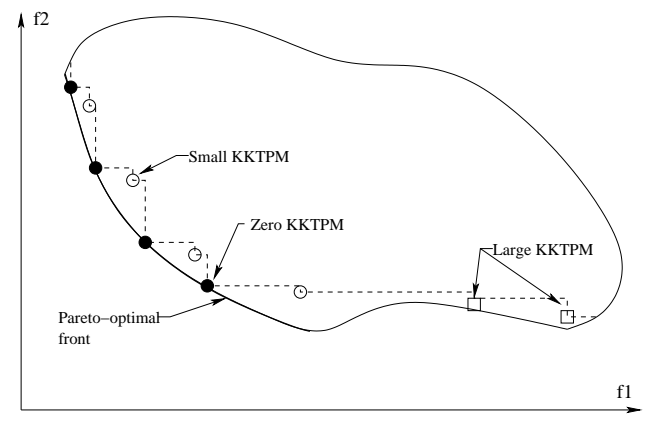

Fig. 2: A set of non-dominated points may have widely different KKTPM values.

Where

$$
\begin{aligned}
& G_{j}(\mathbf{y})=\left(\frac{f_{j}(\mathbf{x})-z_{j}}{w_{j}}\right)+\rho \sum_{j=1}^{M}\left(\frac{f_{j}(\mathbf{x})-z_{j}}{w_{j}}\right)-x_{n+1} \leq 0, \\
& j=1,2, \ldots, M, \text { and } \\
& G_{M+j}(\mathbf{y})=g_{j}(\mathbf{x}) \leq, \quad j=1,2, \ldots, J .
\end{aligned}
$$

The above KKTPM optimization problem needs derivative information of both objectives and constraints functions at each point, meaning that the procedure computationally to find exact KKTPM value at each point is expensive and then takes a long time for any practical use.

Direct KKT proximity metric is an approximate and computationally fast method with exact the same accuracy for the exact KKTPM. This metric has three different approximation procedures (direct $\epsilon_{k}^{D}$, projected $\epsilon_{k}^{P}$ and estimated $\epsilon_{k}^{e s t}$ KKTPM computation approaches) for KKTPM computation. Details of the computational procedures for both the direct, projected and estimated KKTPM methods in [8]. The second study [8] showed surprising results, an estimated $\epsilon_{k}^{e s t}$ KKTPM value are very close to the exact KKTPM value $\epsilon_{k}$. For more detailed results on exact and direct KKT proximity measures, please refer to the original studies [7], [8].

On a number of MOPs, the exact KKTPM metric introduced many beneficial aspects, we mentioned here some of these properties as following [7] 8]:

(1) First, a solution nearer to the strictly PO front has a smallest KKTPM value, in this manner giving a relatively monotonic normal for the KKTPM surface on the objective space.

(2) For every feasible solution, The KKTPM value is always bounded in $[0,1]$.

(3) It is essential to understand that not all non-dominated solutions are probably going to be near PO front. probably going to be near the PO front. Figure 2 shows the following:

-For true PO solutions (marked by filled circles), KKTPM value will be exactly to be zero.

-For all non-dominated solutions that are near to PO front (marked by open circles), KKTPM value will be small.

-For far away non-dominated solutions (marked by open boxes), KKTPM value will be large.

(4) Non-weak PO solutions have good KKTPM values than weak PO solutions.

(5) Non-dominated solutions that have poorly convergence can be identified using KKTPM value and then improves it by using an ASF based local search operator [1].

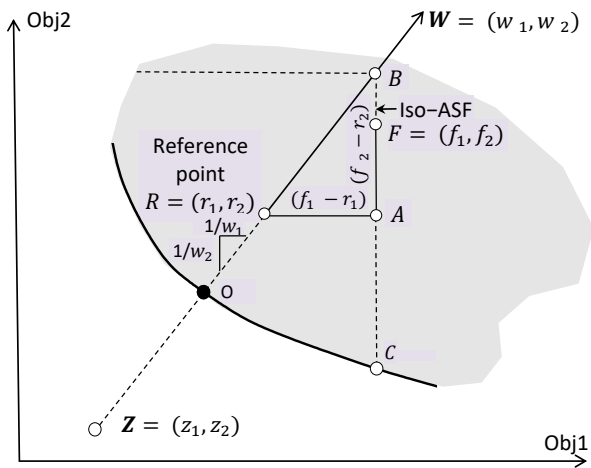

Fig. 3: ASF procedure of finding PO solution for reference point inside the feasible objective space (not utopian point) is illustrated.

(6) From all of the above mentioned properties, KKTPM used for a termination conditions for any multi-objective evolutionary algorithms instead of running the algorithm a fixed number of generation which may not be required.

The following section is presented simulation results using the above KKTPM values and AASF values for ZDT test problems. But before that, some special cases are discussed for each of the measures (exact and direct KKTPM) which show the amazing advantages of these measures

\section{RESULTS AND DISCUSSIONS}

As mentioned above in Section 2, the ASF or AASF values rely on the exact location of the reference point $\mathbf{z}$. Hence, what occurs when change the reference point at PO solutions? Below, an explanation of this case is provided.

(1) An interesting aspect would be when the reference point is taken $R=\left(r_{1}, r_{2}\right)$ inside the feasible objective space (not the utopian point) as shown in Figure 3 then AASF will produce negative value for the respective PO solution, but KKTPM value will be zero at the same reference point $R$.

Table 1 shows exact KKTPM values, direct KKTPM values, and AASF values for two random optimum points for ZDT2 and ZDT4 at different reference points $R=\left(r_{1}, r_{2}\right)$ inside the feasible objective space and under PO front. For example, first, a random optimal solution is taken for ZDT2 problem (suppose that $x_{1}^{*}=0.3876554, x_{i}^{*}=$ 0 for all $i=2,3, \ldots, 30$ ), and with specific weight vector $\frac{1}{w}=[2.409008161529888 ; 1.099176656292937]$. second, a random optimal point is taken for ZDT4 problem (suppose that $x_{1}^{*}=0.5979065$ and $x_{i}^{*}=0$, for all $i=2,3, \ldots, 30)$ with specific weight vector $\frac{1}{w}=$ [1.069536852812447; 2.819367284100803$]$. In both problems, AASF values are negative at reference points that lie inside the feasible objective space, while AASF values are positive at reference points that lie under the PO front. At any reference point, exact and direct KKTPM values are close to zero (at any optimum point). Thus, can be said that KKTPM value, being close to zero, always indicates closeness to the optimum solution. Hence, at any point of reference, KKTPM always ensures that each PO solution has zero value. 
Table 1. : The augmented ASF, exact and direct KKTPM values are illustrated. For number of random reference points that lie inside the feasible objective space and under PO front with specific weight vectors and random optimal solutions for ZDT2 and ZDT4 problems, respectively.

\begin{tabular}{c|cccc}
\hline \multirow{7}{*}{ ZDT2 } & $\begin{array}{c}\text { Reference } \\
\text { points }\end{array}$ & $\begin{array}{c}\text { Exact } \\
\text { KKTPM }\end{array}$ & $\begin{array}{c}\text { Direct } \\
\text { KKTPM }\end{array}$ & $\begin{array}{c}\text { Augmented } \\
\text { ASF value }\end{array}$ \\
\cline { 2 - 5 } & {$[-0.0001 ;-0.0001]$} & 0.000000 & 0.000000 & 0.935974 \\
& {$[0.5 ; 1.095799564]$} & 0.000041 & 0.000000 & -0.271022 \\
& {$[0.2 ; 0.4383913073]$} & 0.000017 & 0.000000 & 0.453031 \\
& {$[1 ; 2.191479992]$} & 0.000082 & 0.000000 & -1.477778 \\
& {$[0.456693776 ; 1.001]$} & 0.000009 & 0.000000 & -0.166612 \\
\hline \multirow{5}{*}{ ZDT4 } & {$[-0.0001 ;-0.0001]$} & 0.000000 & 0.000000 & 0.640869 \\
& {$[0.3 ; 0.1137639046]$} & 0.000021 & 0.000000 & 0.319259 \\
& {$[1.317964847 ; 0.5]$} & 0.000090 & 0.000000 & -0.771669 \\
& {$[1.317964847 ; 0.5]$} & 0.000034 & 0.000000 & 0.104924 \\
& {$[0.8 ; 0.3034738423]$} & 0.000055 & 0.000000 & -0.216579 \\
\hline
\end{tabular}

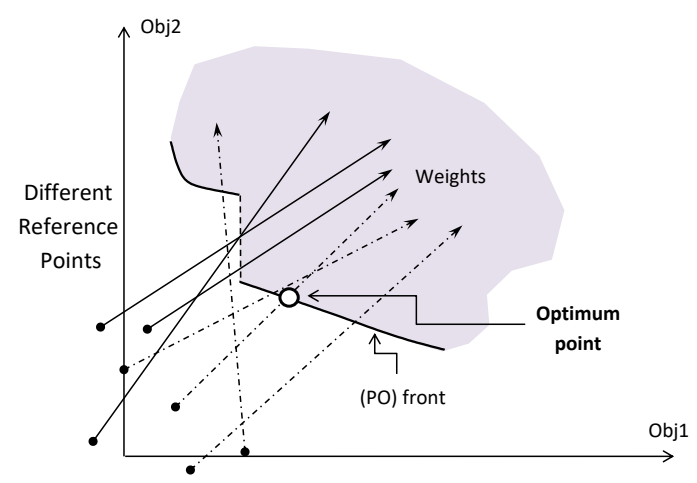

Fig. 4: Weight vectors are such that some of them not intersect with the PO front are illustrated.

(2) Another case would be when the reference and weight vectors are such that the weight vector does not intersect with the PO front. In this case, AASF value will be non-zero, while KKTPM value will be zero for the PO point. Figure 4 illustrates this case. For example, a random optimal point is taken for ZDT3 $\left(x_{1}^{*}=0.4460650\right.$ and $x_{i}^{*}=0$, for $\left.i=2,3, \ldots, 30\right)$, with reference point $z=(0.4,-0.25)$ and weight vector $\frac{1}{w}=$ [3.188303073010045; 1.053141707419629$]$ (this weight vector does not intersect with the PO front). Here, The value of AASF is 0.147163 , while KKTPM value is zero.

(3) Since KKTPM formulation is based on AASF approach, KKTPM value will not be zero for weak PO solutions. In this case also, AASF value will be non-zero. But in strictly PO solution case, KKTPM value will be zero, while AASF value will be non-zero. For example, if a random point is taken on weak efficient front for ZDT2 problem $\left(x_{1}=0, x_{2}=x_{3}=0.9\right.$ and $x_{i}=$ 0 for all $i=4,5, \ldots, 30)$. Thus, KKTPM value for this point is 0.106178 (non-zero) and AASF value is 1.561838 . Also, if a random optimal point is taken on the strict efficient front for the same problem (for example $x_{1}^{*}=0.476065, x_{i}^{*}=0$ for all $i=2,3, \ldots, 30)$. Then, KKTPM value is zero and AASF value is 0.910099 for the same $\mathrm{PO}$ point.

Based on the above properties, KKTPM value will be zero for the PO solution. While, AASF minimum value is unpredictable for the PO solution. All the above advantages for KKTPM show the extent flexible of this metric. In the next subsection, the correlation plot

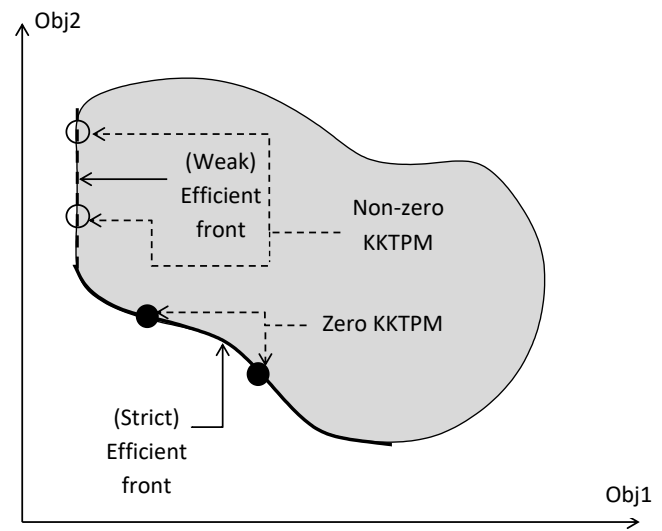

Fig. 5: KKTPM values for weak and strict efficient fronts are illustrated.

between exact KKTPM values and AASF values are investigated, also between direct KKTPM values and AASF values with the specific weight vector and reference point. The aim of this work, if there is a perfect correlation, can be used ASF approach as a metric and no need for KKT proximity measure.

\subsection{Two-Objective ZDT Problems}

The concentrate is only on a reference point based approach for finding a single PO solution. Let us fix a reference point (utopian) and a weight vector. Afterthat, 1000 random points are taken in $\mathrm{x}$ space, and each of them calculates the KKTPM value for a specific weight vector and reference point. Thereafter, The AASF value for each of these points for the same weight and reference vectors is computed. A correlation plot between exact KKTPM values and AASF values is created, also between direct KKTPM values and AASF values. Table 2 shows weights for one random point of 1000 random points for ZTD test problems, which will be used as specific weights.

Table 2. : Specific weights to ZDT test problems for one random point.

\begin{tabular}{c|cc}
\hline \multirow{2}{*}{ ZDT problems } & \multicolumn{2}{|c}{ weight } \\
\cline { 2 - 3 } & $\frac{1}{w_{1}}$ & $\frac{1}{w_{2}}$ \\
\hline ZDT1 & 4.119836471954109 & 1.030827551552600 \\
ZDT2 & 6.486074915527206 & 1.012101361711274 \\
ZDT3 & 5.024358032770250 & 1.020415123125383 \\
ZDT4 & 357.8956472489180 & 1.000003903549700 \\
ZDT6 & 5.546826981150847 & 1.016658212910996 \\
\hline
\end{tabular}

First, ZDT1 problems has 30 variables and convex efficient front. In this problem, all the variables lie in the range $[0,1]$. The efficient solutions occur for $0 \leq x_{1}^{*} \leq 1$ and $x_{i}^{*}=0$ for $i=2,3, \ldots, 30$. Figure 6 shows the relationship between exact (or optimal) KKTPM values $\epsilon_{k}^{*}$ and AASF values, where 1,000 random points were taken in $\mathrm{x}$-space in the range $[0,1]$. After that, a specific weight is taken from Table 2 and specific reference point (utopian). Thereafter, for each of them, the exact KKTPM values are computed for the specific weight vector and reference point, and the AASF values for each of these points are computed for the same weight and reference vectors. It's clear that there is no a perfect correlation between exact KKTPM and AASF values, as evident from Figure 6 


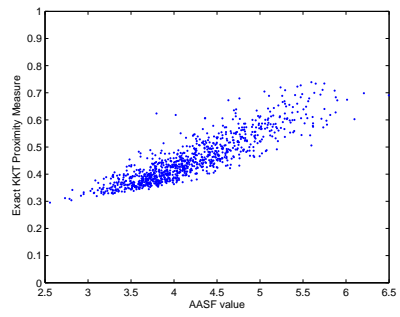

Fig. 6: Correlation plot between exact KKTPM and AASF values to 1,000 random points for problem ZDT1.

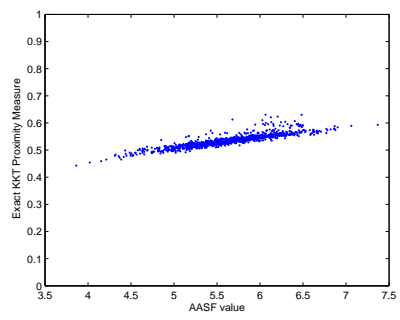

Fig. 8: Correlation plot between exact KKTPM and AASF values to 1,000 random points for problem ZDT2.

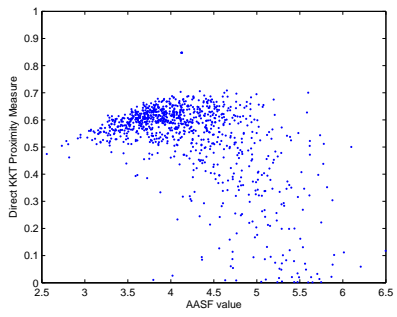

Fig. 7: Correlation plot between direct KKTPM and AASF values to 1,000 random points for problem ZDT1.

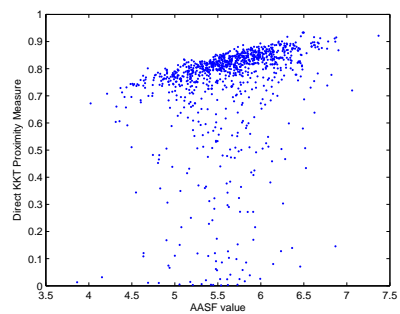

Fig. 9: Correlation plot between direct KKTPM and AASF values to 1,000 random points for problem ZDT2.
Figure 7 shows the relationship between direct KKTPM values and AASF values, the same 1,000 random points are taken in $\mathrm{x}$-space and the same specific weight vector and reference point as $\epsilon_{k}^{*}$ case . Thereafter, for each of them, the direct KKTPM values and AASF values are computed for the same specific weight vector and reference vectors. Figure 7 shows that there is no exact correlation between direct KKTPM and AASF values.

Next, ZDT2 problem has 30 -variables and non-convex PO front. All the variables lie in the range $[0,1]$. The same random points for ZDT1 problem are used with a special weight from Table 2 and reference point (utopian). Figure 8 shows the relationship between exact KKTPM values $\epsilon_{k}^{*}$ and AASF values for ZDT2 problem. For 1,000 random points, the exact KKTPM values for the specific weight vector and reference point are calculated. Also, for the same weight and reference vectors, AASF values for each of these points are calculated. This figure in general shows that increasing the value of AASF then exact KKTPM grows more slowly.

Figure 9 shows the relationship between direct KKTPM values and augmented ASF values for ZDT2 problem to the same specific weight vector and reference point in optimal KKTPM for problem ZDT2. The same situation as ZDT1 problem above, its clear that there is no correlation between exact KKTPM and AASF values, as well as between direct KKTPM values and AASF values. So in general, there is not sufficient relationship.

The ZDT3 problem has 30-decision variables with disconnected PO front. Special weight is taken for one of 1000 random points from Table 2, and reference point. With the special weight and reference points, AASF values are computed for each of these random points. Also, both exact and direct KKTPM values are computed for the special weight and reference points, respec-
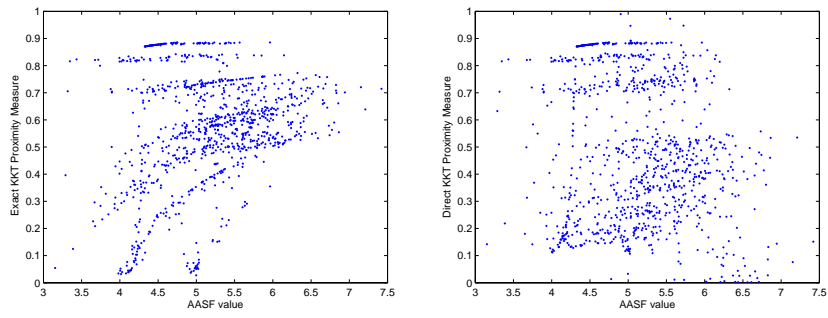

Fig. 10: Correlation plot between exact KKTPM and AASF values to 1,000 random points for problem ZDT3.

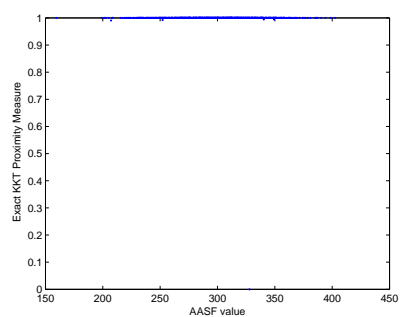

Fig. 12: Correlation plot between exact KKTPM and AASF values to 1,000 random points for problem ZDT4.

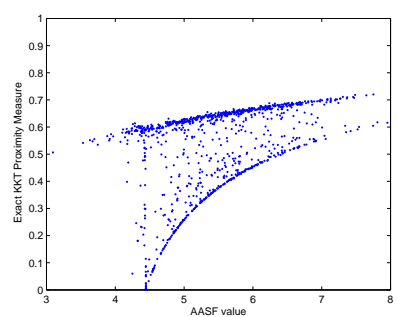

Fig. 14: Correlation plot between exact KKTPM and AASF values to 1,000 random points for problem ZDT6.

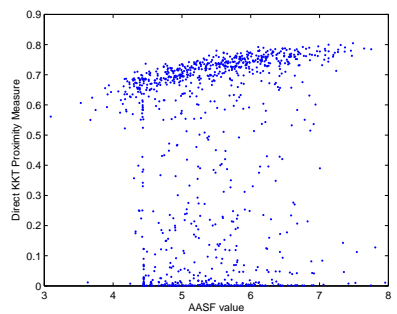

Fig. 15: Correlation plot between direct KKTPM and AASF values to 1,000 random points for problem ZDT6.

tively. In both Figures 10 and 11 show that there is no perfect correlation between exact KKTPM values and AASF values and also between direct KKTPM values and AASF values, respectively.

The 30-variable ZDT4 problem is multimodal and has about 100 local PO fronts. With special weight for this problem (Table 2) and with the same procedures as in above test problems. Figures 12 and 13 shows the relationship between both exact and direct KKTPM values and AASF values respectively. From these figures, its clear that there is no perfect correlation plot between exact KKTPM values and AASF values, and also between direct KKTPM values and AASF values. In this problem, that most the random points are far way from the efficient front as shown in Figures 12 and 13 


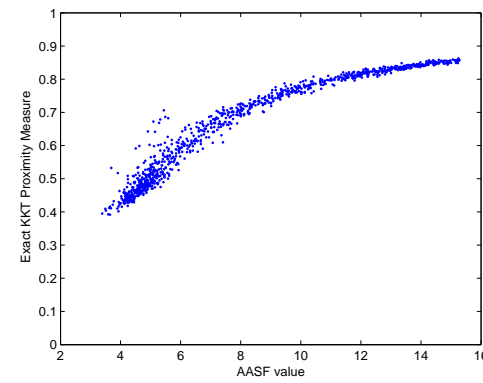

Fig. 16: Correlation plot between exact KKTPM and AASF values to 1, 000 random points for problem ZDT1 with another specific weight vector.

Now, the 10-variable ZDT6 problem has a non-convex PO set. All variables lie in the range $[0,1]$. As the same previous problems, As the same previous problems, the specific weight vector is taken from Table 2 The exact KKTPM, direct KKTPM and AASF values are computed for 1,000 random points. It can be observed that there is no perfect correlation between exact KKTPM values and AASF values, also between direct KKTPM values and AASF values, as shown in Figures 14 and 15 respectively.

Finally, if another specific weight vector is taken for the same 1,000 random points for any ZDT problem. For example ZDT1 test problem, another specific weight vector is $\frac{1}{w}=$ $[15.247832629570404 ; 1.002157533327201]$ With the same reference point (utopia) previously used for ZDT1 problem. Figure 16 shows changes in the value of augmented ASF. Here, AASF value is large but the values of KKTPM are not changed. This change can be seen by comparing between Figures 6 and 16

\section{CONCLUSIONS}

The relationship between KKTPM and AASF values was studied in this paper. The aim of this study was to know the advantages and disadvantages of both measures. KKTPM can be used as metric to know if we have a set of solutions how we are close to the true PO solutions without any given knowledge about these efficient PO set. One of the most important advantages is that KKTPM can be used as a termination criterion to optimization algorithms, such as evolutionary multi-objective optimization methods. Since the KKTPM metric for MOPs based on AASF approach which guarantees to find any PO solution, some special cases were discussed to know the advantages of both the KKTPM and AASF approaches. By studying these special cases, it was observed that approximate KKT proximity measure is an essential measure of convergence. Also, in this work a correlation plot between KKTPM values and AASF values was investigated for ZDT test problems. The aim of this study if there was a perfect correlation, the AASF approach can be used as a metric and KKTPM is no need. Through this study, a perfect correlation between KKTPM values and AASF values cannot be obtained, therefore, AASF cannot be used as a metric. Hence, can be said that KKT proximity measure is better.

\section{REFERENCES}

[1] Mohamed Abouhawwash, Haitham Seada, and Kalyanmoy Deb. Towards faster convergence of evolutionary multicriterion optimization algorithms using karush kuhn tucker optimality based local search. Computers \& Operations Research, 79:331-346, 2017.

[2] Roberto Andreani, José Mario Martínez, and Benar Fux Svaiter. A new sequential optimality condition for constrained optimization and algorithmic consequences. SIAM Journal on Optimization, 20(6):3533-3554, 2010.

[3] Johannes Bader, Kalyanmoy Deb, and Eckart Zitzler. Faster hypervolume-based search using monte carlo sampling. In Multiple Criteria Decision Making for Sustainable Energy and Transportation Systems, pages 313-326. Springer, 2010.

[4] Dimitri P Bertsekas, Angelia Nedi, Asuman E Ozdaglar, et al. Convex analysis and optimization. 2003.

[5] Ş İlker Birbil, Johannes Bartholomeus Gerardus Frenk, and Georg J Still. An elementary proof of the fritz-john and karush-kuhn-tucker conditions in nonlinear programming. European journal of operational research, 180(1):479-484, 2007.

[6] Lam Thu Bui, Slawomir Wesolkowski, Axel Bender, Hussein A Abbass, and Michael Barlow. A dominance-based stability measure for multi-objective evolutionary algorithms. In Evolutionary Computation, 2009. CEC'09. IEEE Congress on, pages 749-756. IEEE, 2009.

[7] K. Deb and M. Abouhawwash. An optimality theory-based proximity measure for set-based multiobjective optimization. IEEE Transactions on Evolutionary Computation, 20(4):515528, Aug 2016.

[8] K. Deb, M. Abouhawwash, and H. Seada. A computationally fast convergence measure and implementation for single, multiple-, and many-objective optimization. IEEE Transactions on Emerging Topics in Computational Intelligence, 1(4):280-293, Aug 2017.

[9] Kalyanmoy Deb and Himanshu Jain. An evolutionary manyobjective optimization algorithm using reference-point-based nondominated sorting approach, part i: Solving problems with box constraints. IEEE Trans. Evolutionary Computation, 18(4):577-601, 2014.

[10] Joydeep Dutta, Kalyanmoy Deb, Rupesh Tulshyan, and Ramnik Arora. Approximate kkt points and a proximity measure for termination. Journal of Global Optimization, 56(4):14631499, 2013.

[11] Michael Emmerich, André Deutz, and Nicola Beume. Gradient-based/evolutionary relay hybrid for computing pareto front approximations maximizing the s-metric. In International Workshop on Hybrid Metaheuristics, pages 140156. Springer, 2007.

[12] Gabriel Haeser and María Laura Schuverdt. On approximate kkt condition and its extension to continuous variational inequalities. Journal of Optimization Theory and Applications, 149(3):528-539, 2011.

[13] H. W. Kuhn and A. W. Tucker. Nonlinear programming. In Proceedings of the Second Berkeley Symposium on Mathematical Statistics and Probability, pages 481-492, Berkeley, Calif., 1951. University of California Press.

[14] Luis Martí, Jesús García, Antonio Berlanga, and José M Molina. An approach to stopping criteria for multi-objective optimization evolutionary algorithms: The mgbm criterion. In Evolutionary Computation, 2009. CEC'09. IEEE Congress on, pages 1263-1270. IEEE, 2009.

[15] Kaisa Miettinen. Nonlinear multiobjective optimization, volume 12. Springer Science \& Business Media, 2012. 
[16] Yury Nikulin, Kaisa Miettinen, and Marko M Mäkelä. A new achievement scalarizing function based on parameterization in multiobjective optimization. OR spectrum, 34(1):69-87, 2012.

[17] A Ravindran, Gintaras Victor Reklaitis, and Kenneth Martin Ragsdell. Engineering optimization: methods and applications. John Wiley \& Sons, 2006.

[18] Ralph Tyrell Rockafellar. Convex analysis. Princeton university press, 2015.

[19] Rupesh Tulshyan, Ramnik Arora, Kalyanmoy Deb, and Joydeep Dutta. Investigating ea solutions for approximate kkt conditions in smooth problems. In Proceedings of the 12th annual conference on Genetic and evolutionary computation, pages 689-696. ACM, 2010.

[20] Tobias Wagner, Heike Trautmann, and Luis Martí. A taxonomy of online stopping criteria for multi-objective evolutionary algorithms. In $E M O$, volume 11, pages $16-30$. Springer, 2011.

[21] Lyndon While, Philip Hingston, Luigi Barone, and Simon Huband. A faster algorithm for calculating hypervolume. IEEE transactions on evolutionary computation, 10(1):2938, 2006.

[22] Andrzej P Wierzbicki. The use of reference objectives in multiobjective optimization. In Multiple criteria decision making theory and application, pages 468-486. Springer, 1980.

[23] Eckart Zitzler, Kalyanmoy Deb, and Lothar Thiele. Comparison of multiobjective evolutionary algorithms: Empirical results. Evolutionary computation, 8(2):173-195, 2000. 